Gut, 1985, 26, 237-240

\title{
Duodenal mucosa synthesis of prostaglandins in duodenal ulcer disease
}

\author{
K HILliER, C L SMITH, R JEWELL, M J P ARTHUR, AND G ROSS \\ From the Clinical Pharmacology Group and Medicine II, Faculty of Medicine, University of Southampton, \\ Southampton
}

Summary Synthesis of prostaglandins $\left(\mathrm{PGE}_{2}, \mathrm{PGI}_{2}\right.$ and $\left.\mathrm{PGF}_{2 \alpha}\right)$ and thromboxane $\mathrm{A}_{2}$ was investigated in short term incubates of duodenal mucosa biopsies. Mucosa close to the ulcer site synthesised significantly less $\mathrm{PGF}_{2 \alpha}(\mathrm{p}<0.001)$ and $\mathrm{PGI}_{2}(\mathrm{p}<0.002)$ measured as its stable metabolite 6-oxo-PGF ${ }_{1 \alpha}$ than healthy mucosa from non-ulcer patients. In paired biopsies taken from the ulcer site and opposite the ulcer in the same patient $\mathrm{PGF}_{2 \alpha}$ and $\mathrm{PGI}_{2}$ syntheses were both significantly and similarly depressed when compared with normal mucosa. Synthesis of $\mathrm{PGE}_{2}$ and $\mathrm{TxA}_{2}$ (as its stable metabolite $\mathrm{TxB}_{2}$ ) was not different in any tissue. There is a defect in the ability of the human duodenal mucosa in duodenal ulcer disease to synthesise PGF $_{2 \alpha}$ and $\mathrm{PGI}_{2}$; the defect is not limited to the ulcer site.

Investigations into the pathogenesis of peptic ulceration and intestinal mucosal injury produced by non-steroidal anti-inflammatory drugs and other damaging agents such as alcohol suggest that the prostaglandins maintain mucosal integrity and accelerate ulcer healing. ${ }^{1-4}$

The mechanisms underlying these beneficial actions are uncertain but studies (mainly with $\mathrm{PGE}_{2}$ ) have shown that it can increase mucus ${ }^{5-6}$ and bicarbonate ${ }^{7}$ secretion. $\mathrm{PGE}_{2}$ can also inhibit acid secretion, ${ }^{4}$ increase gastric mucosal blood flow ${ }^{8}$ and prevent ion flux and potential difference changes produced by noxious stimuli. ${ }^{-11}$

Despite this substantial knowledge of the capabilities of $\mathrm{PGE}_{2}$, information of the actual synthesis and content of $\mathrm{PGE}_{2}$ and other cyclooxygenase derived metabolites (eicosanoids) in gastric and duodenal mucosa of ulcer patients is limited. Moreover, there are several discordant results (see Discussion) and it is probable that extrapolation of results from patients with gastric ulcers will not be valid for duodenal ulceration because of the differences in the types and quantities of prostaglandins synthesised along the gastrointestinal tract. In this study we have examined the synthesis of the prostaglandins (PG), $\mathrm{PGE}_{2}, \mathrm{PGF}_{2 \alpha}$, $\mathrm{PGI}_{2}$ (as its stable metabolite 6-oxo-PGF ${ }_{1 \alpha}$ ) and

Address for correspondence: Dr K Hillier, Faculty of Medicine, Medical and Biological Sciences Building. Bassett Crescent East, Southampton SO9 3TU. Received for publication 18 May 1984 thromboxane $A_{2}$ (as its stable metabolite $\mathrm{TxB}_{2}$ ) in duodenal mucosa.

\section{Methods}

DUODENAL MUCOSAL BIOPSIES

Duodenal mucosa was obtained from 32 subjects with active duodenal ulceration and from 27 subjects undergoing upper gastrointestinal endoscopy for abdominal symptoms later ascribed to irritable bowel. In subjects with duodenal ulcers, the biopsies were taken from within $3 \mathrm{~mm}$ of the ulcer and in subjects without ulceration, from the duodenal cap. In 19 patients two biopsies were taken: one close to the ulcer site and another from the duodenal cap directly opposite the ulcer. The biopsies were frozen immediately in liquid nitrogen and stored at $-70^{\circ} \mathrm{C}$ until analysis. No analyses were carried out on fresh tissue and all samples were treated similarly. Subjects taking drugs liable to affect the upper gastrointestinal tract, particularly ulcer healing preparations or non-steroidal anti-inflammatory drugs were excluded from the study.

This study was approved by the Southampton District Ethical Committee and informed consent obtained.

\section{ASSAY PROCEDURES}

Biopsy specimens weighing between $1-20 \mathrm{mg}$ (average $4.5 \mathrm{mg}$ ) were thawed, weighed, and $2 \mathrm{ml}$ Krebs solution $\mathrm{pH} 7.5$ was added. The solution had 
previously been saturated with $5 \%$ carbon dioxide in oxygen and kept at $4^{\circ} \mathrm{C}$. Specimens were then pre-incubated at $37^{\circ} \mathrm{C}$ for 15 minutes and the supernatant discarded in order to minimise measurements of prostaglandins produced by the trauma of the biopsy procedure.

Fresh Krebs $(2 \mathrm{ml})$ was then added and incubated at $37^{\circ} \mathrm{C}$ for 60 minutes. The supernatant was separated for extraction and the tissue stored at $-20^{\circ} \mathrm{C}$ until protein determination. Approximately $7000 \mathrm{dpm} 9--^{3} \mathrm{H}$ prostaglandin $\mathrm{F}_{2 \alpha}$ (approx $65 \mathrm{pg}$ ) was then added as an internal standard to assess procedural losses. The supernatant was acidified with IM citric acid to $\mathrm{pH}$ 3-4 and immediately extracted twice by mechanical shaking with three volumes distilled diethyl ether and with centrifugation to separate the aqueous and organic phases. The ether was evaporated to dryness under. nitrogen flow at $35^{\circ} \mathrm{C}$ and the residue reconstituted in $2 \mathrm{ml}$ of $0 \cdot 1 \mathrm{M}$ phosphate buffer $\mathrm{pH} 7 \cdot 5$. To assess procedural losses $0.4 \mathrm{ml}$ was taken and its $9-{ }^{3} \mathrm{H}$ $\mathrm{PGF}_{2 \alpha}$ content measured by scintillation spectrophotometry. The recovery was greater than $95 \%$. Following suitable dilutions $\mathrm{PGE}_{2}, \mathrm{PGF}_{2 \alpha}$, 6-oxo$\mathrm{PGF}_{1 \alpha}$ (stable metabolite of $\mathrm{PGI}_{2}$ ) and $\mathrm{TxB}_{2}$ (stable metabolite of $\mathrm{TxA}_{2}$ ) were measured using selective antibodies which in previous tests had been shown to have low cross reactivities with most other major prostaglandins and their metabolites. $\mathrm{PGE}_{2}$, however, cross-reacts $46 \%$ with $\mathrm{PGE}_{1}$ at the level required to produce a $50 \%$ fall in maximum binding of $\left[{ }^{3} \mathrm{H}\right] \mathrm{PGE}_{2}$ to the antibody in the radioimmunoassay.

The PG or $\mathrm{TxB}_{2}$ content of 0.1 and $0.2 \mathrm{ml}$ of unknown samples was measured and results accepted only if they showed concordance with the standards which were always assessed in parallel. Blank values obtained by passing Krebs solution through the entire procedure were included. In 12 experiments the mean blank values for $0.1 \mathrm{ml}$ of the $2 \mathrm{ml}$ reconstituted extracts were $(\mathrm{pg} \pm \mathrm{SD}) 3 \cdot 0 \pm 2 \cdot 1$ $\left(\mathrm{PGE}_{2}\right), 4 \cdot 0 \pm 2 \cdot 5\left(\mathrm{PGF}_{2 \alpha}\right), 3 \cdot 4 \pm 2 \cdot 9$ (6-oxo-PGF $\left.1 \alpha\right)$ and $2 \cdot 3 \pm 2 \cdot 0\left(\mathrm{TxB}_{2}\right)$. All samples were assayed in duplicate and in four separate samples the PGs and $\mathrm{TxB}_{2}$ were measured in two consecutive assays to assess reproducibility. The results showed good agreement with no significant difference between estimates in the two assays. Because of the small biopsy samples a more extensive evaluation of the reproducibility of the assays was not possible. In studies in this laboratory, however, on PG synthesis by colonic mucosa and using identical methods, the intra-assay coefficient of variation for $\mathrm{PGE}_{2}$ was $9.5 \%(n=8)$ and for $\mathrm{PGF}_{2 \alpha}$ was $9.09 \%(n=7)$. The respective intraassay coefficients of variation were $9.0 \%(n=7)$ and $8.3 \%(n=7)$.
Protein concentrations following digestion with $0 \cdot 1 \mathrm{~N}$ sodium hydroxide were measured by the method of Lowry et al. ${ }^{12}$ Results were analysed by Wilcoxon's rank sum test and Student's $t$ test (paired or unpaired) where appropriate.

The term synthesis is used in this paper to describe the accumulation of eicosanoids in the incubation medium. 6-oxo- $\mathrm{F}_{1 \alpha}$ and $\mathrm{TxB}_{2}$ would not be subject to further metabolic degradation and in the absence of added NAD the metabolism of $\mathrm{PGF}_{2 \alpha}$ and $\mathrm{PGE}_{2}$ would be minimal.

\section{Results}

Table 1 shows the eicosanoids synthesised by healthy, non-ulcerated mucosa in a group of 27 patients without duodenal ulceration compared with biopsies taken close to the ulcer site in 32 separate patients with duodenal ulceration. During 60 minutes incubation, synthesis of $\mathrm{PGF}_{2 \alpha}$ and 6-oxo$\mathrm{PGF}_{1 \alpha}$ by ulcer tissue was reduced by $45.4 \%$ and $47.6 \%$ respectively when compared with that synthesised by control mucosa, whereas $\mathrm{PGE}_{2}$ and $\mathrm{TxB}_{2}$ showed no differences between control and ulcer subjects.

Table 2 compares eicosanoid synthesis in 19 pairs of mucosal biopsy specimens from the same patients taken close to and opposite the ulcer site. The synthesis of all eicosanoids at both sites was not significantly different. Thus synthesis of $\mathrm{PGF}_{2 \alpha}$ and 6-oxo-PGF $F_{1 \alpha}$ in samples taken from opposite the ulcer was also significantly lower than in mucosa from patients without duodenal ulceration by $38.9 \%$ and 53\% respectively $(\mathrm{p}<0.01$ using Wilcoxon's rank sum test and Student's $t$ test).

\section{Discussion}

We have shown highly significantly reduced $\mathrm{PGI}_{2}$ and $\mathrm{PGF}_{2 \alpha}$ synthesis by duodenal mucosa within 3 $\mathrm{mm}$ of ulcer compared with healthy mucosa from

Table 1 Prostanoid and thromboxane synthesis by duodenal mucosa close to the ulcer site compared with mucosa from the duodenal cap in patients without duodenal ulcer

\begin{tabular}{lllcll}
\hline & $N o$ & $P G E_{2}$ & $P G F_{2 \alpha}$ & 6-oxo-PGF $F_{l \alpha}$ & $T x B_{2}$ \\
\hline Control & 27 & $17 \cdot 6 \pm 2 \cdot 75$ & $10 \cdot 8 \pm 1 \cdot 07$ & $17 \cdot 0 \pm 2 \cdot 2$ & $17 \cdot 4 \pm 1 \cdot 5$ \\
Ulcer & 32 & $18 \cdot 8 \pm 2 \cdot 7$ & $5 \cdot 9 \pm 0 \cdot 6 \dagger$ & $8 \cdot 9 \pm 1 \cdot 1^{*}$ & $17 \cdot 9 \pm 1 \cdot 8$ \\
\hline
\end{tabular}

Values shown are $\mathrm{ng} / \mathrm{mg}$ protein $\pm \mathrm{SEM}$ of eicosanoid released into the incubation fluid in one hour. The values shown in the ulcer row include the 19 samples from close to the ulcer site shown in Table 2 Statistical analysis was performed using Wilcoxon's rank sum test. ${ }^{*} p<0.002$ and $+p<0.001$.

By Student's $t$ test ${ }^{*} \mathrm{p}<0.001$ and $* \mathrm{p}<0.001$. 
Table 2 Prostanoid and thromboxane synthesis by duodenal mucosa close to the ulcer site and from the duodenal cap directly opposite the ulcer

\begin{tabular}{|c|c|c|c|c|c|}
\hline & No & $P G E_{2}$ & $P G F_{2 \alpha}$ & 6-oxo-PGF $F_{I a}$ & $T \times B_{2}$ \\
\hline Close to ulcer site & 19 & $14 \cdot 2 \pm 2 \cdot 4$ & $4 \cdot 8 \pm 0 \cdot 6$ & $8 \cdot 0 \pm 1 \cdot 3$ & $17 \cdot 0 \pm 2 \cdot 15$ \\
\hline Opposite ulcer site & 19 & $15 \cdot 8 \pm 3 \cdot 9$ & $6 \cdot 6 \pm 0 \cdot 7$ & $8 \cdot 0 \pm 1 \cdot 3$ & $17 \cdot 0 \pm 2 \cdot 1$ \\
\hline
\end{tabular}

Data shown are paired values ( $\mathrm{ng} / \mathrm{mg}$ protein $\pm \mathrm{SEM}$ ) of eicosanoid released into the incubation fluid in one hour. The eicosanoids synthesised in biopsies from two sites in the same patient were measured in the same assay. Statistical analysis was performed using Wilcoxon's rank sum test and by Student's $t$ test.

non-ulcerated patients. Further, the synthesis of eicosanoids in the samples of mucosa from opposite the ulcer site are similarly reduced when compared with mucosa close to the ulcer. It would seem, therefore, that the prostanoid synthesising defect is not just associated with the immediate ulcer area but is a more general duodenal abnormality in ulcer disease and not secondary to the formation of the ulcer.

Comparison of our data with other investigations highlights some dissimilarities. In a similar study to this but utilising different culture conditions Sharon et $a l^{13}$ showed no reduction in 6 -oxo-PGF $1 \alpha$ accumulation in culture fluid of incubated duodenal mucosa from ulcer patients; however, like this study they showed no diminished $\mathrm{PGE}_{2}$ or $\mathrm{TxB}_{2}$ accumulation. Other investigators also showed no change in $\mathrm{PGE}_{2}$ synthesis in the duodenum from patients with ulcers compared with healthy duodenal mucosa. ${ }^{14}$

Evidence for a more general mucosal defect in eicosanoid synthesis has been suggested ${ }^{11}$ as seemingly healthy gastric mucosa from patients with duodenal ulceration synthesised less $\mathrm{PGE}_{2}$, 6-oxo$\mathrm{PGF}_{1 \alpha}$ and $\mathrm{TxB}_{2}$ than gastric mucosa from patients without duodenal disease. ${ }^{13}$ Other investigators, however, could not show this. ${ }^{14}$ Further uncertainties exist as other investigators studying tissue content of $\mathrm{PGE}_{2}$ have shown reduced concentrations in mucosa both at the edge of and $5 \mathrm{~cm}$ from the gastric ulcer site compared with normal tissue. ${ }^{15}$ Contrary to this, raised concentrations of prostanoids in antral biopsies from gastric ulcer patients $(4 \mathrm{~cm}$ from the lesion) compared with controls have also been described. ${ }^{16}$ These discrepancies may be because of methodological differences or to variations in the site of biopsy. We believe that these may be resolved by more clearly defining the region from which the biopsy is taken and by realising that there will be marked differences between the ability of tissue to newly synthesise prostanoids and thromboxane when compared with the tissue content and, because cells do not contain storage sites for prostaglandins the measurement of tissue content may not be meaningful.

We were able to show reduced levels of synthesis of only $\mathrm{PGI}_{2}$ and $\mathrm{PGF}_{2 \alpha}$ in ulcer, patients; this selectivity is perhaps surprising because $\hat{\mathrm{PGE}}_{2}$ in also cytoprotective. ${ }^{17}$ As $\mathrm{PGE}_{2^{\prime}}$ and $\mathbf{T} \mathbf{x} \mathbf{A}_{2}$ synthesis is maintained in ulcer tissue it is unlikely that there is an overall reduction in cyclooxygenase enzyme activity or substrate availability although we cannot yet be certain of this. The nature of the reduced synthesis suggests that dissimilar cell types may be responsible for the different PGs synthesised and these are altered in ulcer disease. The significance of $\mathrm{TxA}_{2}$ in peptic ulcer disease is not known; it is vasoconstrictor and platelet aggregatory and as such may exacerbate mucosal damage; however, PGF $_{2 \alpha}$ can be vasoconstrictor yet also has cytoprotective properties. ${ }^{17}$ The selective reduction in the synthesis of the potent vasodilator $\mathrm{PGI}_{2}$ while maintaining $\mathrm{TxA}_{2}$ may lead to reduced blood flow in the ulcerated duodenal mucosa and contribute to the ulcer or delay healing.

It is possible that the relative balance of the PGs and $\mathrm{TxA}_{2}$ synthesised may be an important factor in the genesis of ulcers as may be the synthesis of the lipoxygenase products of arachidonic acid metabolism.

We conclude that reduced $\mathrm{PGI}_{2}$ and $\mathrm{PGF}_{2 \alpha}$ may predispose the duodenal mucosa to injury and ulceration.

Antisera were generous gifts from Dr J Salmon, Wellcome (6-oxo-PGF ${ }_{1 \alpha}$ ), and $\mathrm{Dr} \mathrm{M}$ Mitchell $\left(\mathrm{TxB}_{2}\right)$. Standard prostaglandins and thromboxane $\mathrm{B}_{2}$ were gifts from Dr $\mathrm{J}$ Pike (The Upjohn Company, USA). We are grateful to the staff of Medicine II for their help in obtaining specimens. We gratefully acknowledge the financial assistance of Glaxo Group Research.

\section{References}

1 Peskar BM, Weiler $\mathrm{H}$, Meyer Ch. Inhibition of prostaglandin production in the gastrointestinal tract by 
anti-inflammatory drugs. Adv Inflammation Res 1984; 6: 40-9.

2 Robert A. Prostaglandins and digestive diseases. $A d v$ Prostaglandin Thromboxane Res 1980; 8: 1533-41.

3 Johansson C, Kollberg B, Nordemar R, Samuelson K, Bergstrom S. Protective effect of prostaglandin $E_{2}$ in the gastrointestinal tract during indomethacin treatment of rheumatic diseases. Gastroenterology 1980; 78: 479-83.

4 Robert A, Schultz JR, Nezamis JE, Lancaster C. Gastric antisecretory and anti ulcer properties of $\mathrm{PGE}_{2}, 15$-methyl $\mathrm{PGE}_{2}$, and 16,16-dimethyl $\mathrm{PGE}_{2}$. Intravenous, oral and intrajejunal administration. Gastroenterology 1976; 70: 359-70.

5 Johansson C, Kollberg B Stimulation by intragastricallv administered $E_{2}$ prostaglandins of human gastric mucus output. Eur J Clin Invest 1979; 9: 229-32.

6 Bolton JP, Palmer D, Cohen MM. Effects of the $E_{2}$ prostaglandins on gastric mucus production in rats. Surg Forum 1976; 27: 402-3.

7 Ross IN, Turnberg LA. Studies of the 'mucusbicarbonate' barrier on rat fundic mucosa: the effects of luminal $\mathrm{pH}$ and a stable prostaglandin analogue. Gut 1983; 24: 1030-3.

8 Gerber JG, Nies AS. Canine gastric mucosal vasodilation with prostaglandins and histamine analogs. Dig Dis Sci 1982; 27: 870-4.

9 Chaudhury TK, Jacobson ED. Prostaglandin cytoprotection of gastric mucosa. Gastroenterology 1978; 74: 58-63.

10 Whittle BJR. Mechanism underlying gastric mucosal damage induced by indomethacin and bile-salts and the actions of prostaglandins. Br J Pharmacol 1977; 60: $455-60$.

11 Tepperman BL, Miller TA, Johnson LR. Effect of 16,16-dimethyl prostaglandin $E_{2}$ on ethanol-induced damage to canine oxyntic mucosa. Gastroenterology 1978; 75: 1061-5.

12 Lowry OH, Rosebrough NJ, Farr AL, Randall RJ. Protein measurement with the folin phenol reagent. $J$ Biol Chem 1951; 193: 265-75.

13 Sharon P, Cohen F, Zifroni A, Karmeli F, Ligumsky M, Rachmilewitz D. Prostanoid synthesis by cultured gastric and duodenal mucosa: possible role in the pathogenesis of duodenal ulcer. Scand J Gastroenterol 1983; 18: 1045-9.

14 Konturek SJ, Obtulowicz N, Sito E, Wilkon S, Dembinska-Kiec A. Distribution of prostaglandins in gastric and duodenal mucosa of healthy subjects and duodenal ulcer patients: effects of aspirin and paracetamol. Gut 1981; 22: 283-9.

15 Wright JP, Young GO, Klaff LJ, Weers LA, Price SK, Marks IN. Gastric mucosal prostaglandin E levels in patients with gastric ulcer disease and carcinoma. Gastroenterology 1982; 82: 263-7.

16 Schlegel W, Wenk K, Dollinger HC, Raptis S. Concentrations of prostaglandin $\mathrm{A}, \mathrm{E}$ and $\mathrm{F}$ like substances in gastric mucosa of normal subjects and of patients with various gastric diseases. Clin Sci Molec Med 1977; 52: 255-8.

17 Robert A. Cytoprotection by prostaglandins. Gastroenterology 1979; 77: 761-7. 